\title{
A new method of measuring the rate of shedding of epithelial cells from the intestinal villus of the rat
}

\author{
RUFUS M. CLARKE ${ }^{1}$ \\ From the Physiological Laboratory, University of Cambridge
}

SUMMARY Epithelial cells shed from the intestinal villus in starved rats were trapped in mucus which the goblet cells were stimulated to secrete; the number of trapped cells per villus was counted in whole mounts of fixed Feulgen-stained specimens from three sites in the small intestine. Increase in the interval between stimulation and fixation caused an increase in the number of shed cells per villus; the rate of this increase was postulated to represent the rate of cell shedding.

The rate of cell production per crypt was measured with Colcemid; this rate, multiplied by the number of crypts per villus, gave the rate of cell production per villus. There was good agreement between these estimates of cell production and cell shedding.

The size of the epithelial cell population of the small intestine remains relatively constant, presumably as a result of the balance between the rate at which cells are produced in the crypts of Lieberkühn and the rate at which they are shed from the villi. Investigation of the nature of this balance would be facilitated if it were possible to measure both of these rates in absolute terms. A method for measuring the rate of cell production has been described (Clarke, 1970a) and validated (Clarke, 1971), and this paper describes a new method of attempting to measure the rate at which cells are shed from the villi.

'Extrusion zones' on the villi were described by Leblond and Stevens (1948) in the rat, and O'Connor (1966) used their presence as an index of cell shedding in the young mouse. It is obvious that the proportion of sectioned villi bearing 'extrusion zones' will depend not only upon the rate of cell shedding, but also on the duration of this process, and it therefore has little quantitative meaning. A different approach was pursued by Loehry, Croft, Singh, and Creamer (1969); the intestinal lumen of an anaesthetized rat was perfused with sodium chloride solution, and the

Received for publication 22 July 1970.

${ }^{1}$ Present address : Department of Human Morphology, University Park, Nottingham NG7 2RD.
DNA content of the perfusate was measured. Since the weight of DNA per rat cell is known, the rate of cell shedding could be calculated.

The method described here arose from an attempt to confirm the findings of Loehry et al (1969). During the course of this investigation, two observations cast doubt on the absolute quantitative validity of the method of Loehry et al (1969): first, that after perfusion of any duration gentle removal of the small intestine from the abdominal cavity caused a considerable increase in the cellularity of the perfusate; and second that, even after this manoeuvre, histological examination of the small intestine showed many epithelial cells trapped in layers of mucus secreted by the goblet cells. It was dubious, therefore, whether such a perfusion resulted in quantitative recovery of the shed cells, and this suspicion was strengthened by Loehry's finding (1968) that increasing the rate of perfusion increased the recovery of DNA in the perfusate. Finally, the rate of cell shedding calculated from the figure of Loehry et al (1969) for DNA recovery in rats which have been starved overnight is about one sixth of the rate of cell production in the whole small intestine of fed rats (Clarke, unpublished observations), although Cotton (personal communication) has modified the method of 
Loehry et al (1969), and obtains values for the rate of cell shedding which are in good agreement with the rate of cell production.

Attempts were therefore made either to abolish the secretion of mucus or to reduce its viscosity; atropinization, the use of various anaesthetic agents, alterations in the composition of the perfusing fluid, and the use of proprietary mucolytic substances all proved ineffective, and it was concluded that the secretion of mucus and the trapping of cells are inescapable responses to perfusion of the intestinal lumen. With this conclusion came the realization that the sheet of mucus might be used to trap all the epithelial cells shed over a short period, thus providing an index of the rate of cell shedding. The experiments to be described suggest that it is indeed possible to measure the rate of cell shedding by this method.

\section{Materials and Methods}

Male albino Wistar rats were obtained from A. Tuck and Son, Rayleigh, Essex, and kept in the laboratory's animal house for at least one week before use at a temperature of $21^{\circ} \mathrm{C}$. Throughout they had free access to tap water, but for four days before the experiment were completely deprived of pellets of diet 41B (Oxoid), to which they had previously had free access. Rats deprived of solid food for four days were used because solid intestinal contents made difficult the perfusion of the small intestine of the fed rat, and because these animals form the baseline for further experiments. During the period of food deprivation, the rats lost $24-30 \%$ of their previous body weight, and weighed $120-170 \mathrm{~g}$ on the day of experiment. A total of 39 rats were used.

MEASUREMENT OF CELL PRODUCTION RATE Thirty rats were injected intraperitoneally with Colcemid (Ciba), $2.5 \mathrm{mg} / \mathrm{kg}$ body weight in water for injection BP, between 8.30 and 11.30 a.m. All these animals were subsequently treated as described below. The interval between the injection of Colcemid and the fixation of the intestine was recorded to the nearest minute.

MEASUREMENT OF CELL SHEDDING RATE This new technique is described in detail, since attention to detail appears to be essential for satisfactory results. All 39 rats were injected intraperitoneally with pentobarbitone sodium BP $60 \mathrm{mg} / \mathrm{kg}$ body weight in water for injection BP. Of these, 19 were treated in the manner described below; the others were used to test modifications of the method which proved to be unsatisfactory. As soon as surgical anaesthesia was achieved, the rat was placed on a warm table, and the stomach exposed through a midline upper abdominal incision. A short polythene tube of diameter $4 \mathrm{~mm}$ was inserted through a small incision in the duodenum, and tied in place with a ligature immediately distal to the opening of the bile duct. The caecum was brought out through the abdominal incision, and a polythene tube of diameter $7 \mathrm{~mm}$ was introduced into the proximal colon and tied in place. The cannulated segments of intestine were replaced in the peritoneal cavity, and the incision was covered with a moist swab. The duodenal cannula was connected to a reservoir of solution maintained at $38^{\circ} \mathrm{C}$, whose composition was sodium chloride $9 \mathrm{~g}$, polyethylene glycol $400010 \mathrm{~g}$, and diaminoethanetetraacetic acid (disodium salt-EDTA) $3 \mathrm{~g}$ per litre of solution in distilled water. The solution was allowed to run into the small intestine under gravity at a pressure of $30 \mathrm{~cm}$ of water, and usually appeared in the outflow cannula within 30 seconds. Any animal in which this took longer than $60 \mathrm{sec}$ was discarded. The outflow tubing was raised $10 \mathrm{~cm}$ above the table to provide a satisfactory distending pressure in the intestine and after the solution had been flowing freely from the intestine for a few seconds, the tubing was clamped close to the colon. Inflow of solution to the intestine was allowed to continue after the outflow had been clamped, and occurred at a rate of 1 drop every 5-10 sec, presumably due to intestinal absorption of some of the solution. There was no appreciable leakage to the peritoneal cavity.

After an accurately timed interval of 0-15 min (described in the results as 'duration of exposure to perfusate'), the outflow clamp was released and simultaneously, by means of a three-way tap, a fixative mixture of absolute ethanol ( 3 parts) and glacial acetic acid (1 part) was allowed to flow in through the duodenal cannula under gravity at a pressure of $33 \mathrm{~cm}$ of mixture. Fixative appeared in the outflow cannula after about $15 \mathrm{sec}$, and was allowed to flow for a further 10-20 seconds. The outflow cannula was again clamped, and the intestine allowed to distend with fixative at the same pressure until inflow had almost ceased. The small intestine was ligated at each end, the cannulae removed, the abdominal incision enlarged, and the small intestine dissected out with minimal disturbance and handling by cutting through the root of the mesentery, and placed in a corked tube containing the same fixative mixture.

After fixation overnight, the small intestine was dissected free of mesentery and flushed out gently with $75 \%$ ethanol from a syringe attached to a needle inserted in the duodenum. Fluid was allowed to escape through another needle in the terminal ileum, to keep the intestine distended. The intestine was then placed in $75 \%$ ethanol for at least 24 hours, until observations were made. 
The intestine was laid out straight, and pieces approximately $0.5 \mathrm{~cm}$ square were cut from the antimesenteric region, at sites $10 \%, 50 \%$, and $95 \%$ of the length of the small intestine from the pylorus. These were treated with especial care in order not to damage the mucosal surface. Hydration in $50 \%$ ethanol and distilled water was followed by hydrolysis in N/1 hydrochloric acid at $60^{\circ} \mathrm{C}$ for $15 \mathrm{~min}$, thorough washing in distilled water, and staining in Schiff's reagent for at least one hour (Wimber and Lamerton, 1963). Specimens were then washed in tap water and transferred to $45 \%$ acetic acid. Each specimen was placed, villi uppermost, on a microscope slide in a pool of $45 \%$ acetic acid and a coverslip was gently applied. On examination with transmitted light at a magnification of $\times 250$ the nuclei of the intact epithelium of the villus could be seen clearly on focusing, and above this layer, in cells entrapped in translucent mucus on the surface of the villus, a group of nuclei in various stages of dissolution could be seen. The number of such extra-epithelial nuclei was counted on 20 villi in each specimen, the small nuclei of lymphocytes being easily recognizable, and excluded from the count. If the cells were obscured by debris, or if the villus epithelium was damaged, with normal nuclei outside the epithelial plane, examination of an adjacent area enabled satisfactory counts to be made.

\section{OTHER MEASUREMENTS}

After this, each specimen was treated as described by Clarke (1970a). Photographs were taken with transmitted light from the serosal aspect, focusing in turn on crypts and villi at magnifications of 20 and 10 respectively. Counts were made from enlargements of these negatives (magnified five times) to obtain estimates of the numbers of crypts and villi per $\mathrm{mm}^{2}$ of serosal area, and from these the crypt/villus ratio was calculated. Under a dissecting microscope individual crypts of Lieberkühn were dissected from each specimen, transferred to a fresh slide, and squashed gently under a coverslip. The number of colchicinemetaphases in each of 10 crypts was counted at a magnification of $\times 400$.
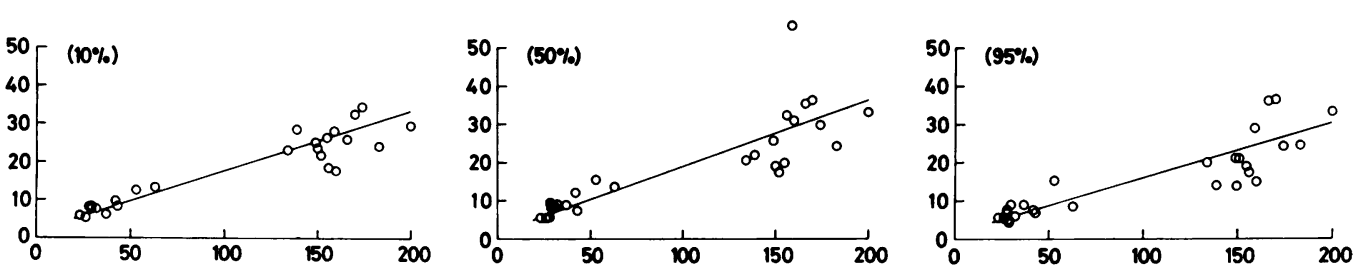

DURATION OF EXPOSURE TO COLCEMID IN MIN
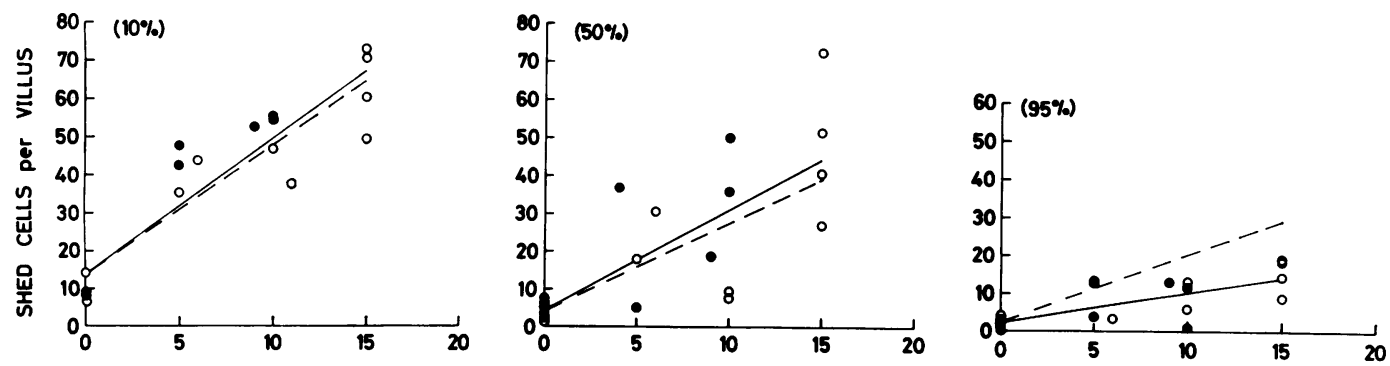

DURATION OF EXPOSURE TO PERFUSATE IN MIN

Fig. Above Accumulation of colchicine-metaphases at three sites in the small intestine of rats starved for four days. The ordinate is mean colchicinemetaphases per crypt (10 crypts per point). Straight lines were calculated by the method of least squares.

Below Accumulation of shed cells in mucus at the top of the villus at the same three sites. The ordinate is mean cells shed per villus (20 villi per point). $\bigcirc=$ animals exposed to Colcemid; $\bigcirc=$ animals not exposed. The solid line was calculated by the method of least squares and represents the rate of cell shedding per villus; the broken line is the rate of cell production per villus. 


\section{Results}

CRYPT/VILLUS RATIO

The number of crypts per villus was $21.9 \pm 2.9$ (mean and SD of 36 observations) at $10 \%$ from the pylorus, $14.9 \pm 2.3$ at $50 \%$, and $12.4 \pm 1.9$ at $95 \%$. These values are not statistically significantly different (Student's $t$ test) from the values for fed rats (Clarke, 1970a).

\section{CELL PRODUCTION RATE PER CRYPT}

Anaphases and telophases were seen in three of the 30 rats and these were discarded. The rate of accumulation of colchicine-metaphases in crypts at each of the three sites is shown in the Figure (p. 1017). Each point represents the mean of 10 crypts in one animal; the standard deviation was usually $20-30 \%$ of the mean. As in the fed rat (Clarke, 1970a), there was little difference in the rate at different sites in the intestine. Preliminary experiments showed that the rate was unaffected by anaesthesia and intestinal perfusion during the period of metaphase blockade.

CELL PRODUCTION RATE PER VILLUS

This is the product of the crypt/villus ratio and the cell production rate per crypt. The calculated figures are shown in the Table.

\begin{tabular}{lccc}
\hline & \multicolumn{3}{c}{$\begin{array}{c}\text { Distance from Pylorus (\% of } \\
\text { length }\end{array}$} \\
& 10 & small & intestine) \\
\hline & $9 \cdot 3$ & $10 \cdot 2$ & 95 \\
Cell production rate/crypt/hour & $21 \cdot 9$ & $14 \cdot 9$ & $12 \cdot 4$ \\
Crypt/villus ratio & 204 & 152 & 108 \\
Cell production rate/villus/hour & 214 & 160 & 48 \\
Cell shedding rate/villus/hour & &
\end{tabular}

Table Comparison of the rates of cell production and cell shedding in starved rats

CELL SHEDDING RATE PER VILLUS

It was feasible to count the number of shed cells overlying one villus since, until the number reached about 100 , the number of layers of cells was small. As the duration of exposure to perfusate was increased signs of nuclear disintegration were seen, the appearances being consistent with the following sequence of events: the nuclear membrane became thickened and blebs of chromatin were deposited on it; the membrane then broke up, the blebs remaining in a circular pattern. Finally, the blebs became spherical and broke up into smaller fragments. Clearly at this stage it became impossible accurately to estimate the number of nuclei originally present in the mucus; in starved animals this stage was reached in the proximal intestine after about 20 minutes' exposure to perfusate and counts up to $15 \mathrm{~min}$ were possible. Distally, the process appeared to proceed more slowly (as judged by delay in reaching equivalent stages of degeneration), and longer periods of exposure would be possible distally in starved animals.

In the Figure (p. 1017) the results of these experiments are plotted. Each point represents the mean of 20 counts in one animal; the standard deviation of counts usually lay in the range 30$40 \%$ of the mean. Colcemid appeared to have no effect on the rate of cell shedding. Although there is considerable scatter in some cases, the trend of accumulation of shed cells with time is clear. The two proximal sites show good agreement between the rate of cell shedding and the rate of cell production; there is no obvious explanation for the poorer agreement in the terminal ileum.

\section{Discussion}

The basic experimental manoeuvre used in the estimation of both these rates of cell turnover is the measurement of the rate of accumulation of cells at an artificial block in the natural sequence of events. The use of colchicine in blocking cell division is well known and has been validated (Clarke, 1971). The accumulation of shed cells in mucus has not been described before and the validity of using it as a method of estimating the rate of cell shedding will depend on the extent to which the assumptions inherent in the manoeuvre are fulfilled.

These assumptions are: (1) that the shed celis remain intact, recognizable, and countable in the mucus during the experimental period; (2) that no shed cells escape being trapped in the mucus; (3) that the experimental conditions cause no change in the rate of cell shedding.

The first assumption is borne out by the experience of this investigation; experimental periods up to $15 \mathrm{~min}$ were possible in the proximal intestine of these starved animals, although it may be that in fed animals enzymic activity is greater and a shorter duration will be necessary. Certainly if the rate of cell shedding in fed animals exceeds that in starved animals by the same proportion (three- to four-fold) as does the rate of cell production (Clarke, 1970b), the number of cells shed per villus will become too great to count in a period much less than 15 minutes.

The second assumption is that no shed cells escape from the mucus. If they do so intact, then one would expect either to find them attached to mucus further down the intestine, in which case the rate of cell shedding distally should be higher than predicted, or to find them in the perfusate. Neither of these predictions was fulfilled. If the cells escape from the mucus and disintegrate and vanish without trace then the arguments in the next paragraph apply.

The final assumption is that the rate of cell 
shedding is not affected by the experimental conditions. It might be thought optimistic to suppose that anaesthesia had no effect and that the fluid introduced into the intestinal lumen was sufficiently noxious to cause goblet cell discharge yet benign enough not to alter the rate of cell shedding from the villus. This optimism is justified by the good agreement between the rate of cell production and the rate of cell shedding, except for the unexplained discrepancy in the terminal ileum. The expectation of such agreement implies that, after four days' starvation, epithelial turnover is in a steady state, cell production and shedding being in equilibrium. Since the height of the villi in the proximal intestine is diminished after four days' starvation (Clarke, 1970b), cell shedding probably exceeded cell production at some stage; but since the cell production rate and villus height are the same at four and at five days' starvation (Clarke, unpublished observations) it is probable that a steady state has been regained.

The chelating agent EDTA was originally included in the perfusion fluid on account of its inhibitory effect on desoxyribonuclease (Marmur, 1961), although it might be thought likely to affect the rate of cell shedding in view of the role of calcium ions in cell adhesion (Ringer, 1890). However, nuclear disintegration did not seem to proceed more rapidly in animals perfused with solution lacking EDTA as judged by the degree of nuclear degeneration compared with that in animals perfused with EDTA. Also the counts of cells shed per villus showed greater scatter and a much less obvious trend of increase with time in animals not exposed to EDTA. Since the rate of cell shedding, even with the use of EDTA, shows satisfactory agreement with the rate of cell production it is concluded that the use of EDTA does not alter the rate of cell shedding.

It is thought that this technique, while not claimed to be perfect, might be of interest because it offers advantages over other methods; measurements of DNA in intestinal perfusate relate to the rate of cell shedding in the whole small intestine or in that segment which is perfused (McFarland, Asplin, and Hallaway, 1968; Goldsmith, 1969; Loehry et al, 1969). The advantage of the method described here is that the rate of cell shedding can be measured at individual sites in the intestine; this may be of particular benefit in situations where spatial resolution of changes in the rate of cell shedding is required, for example after intestinal resection.
Coupled with this is the ability to measure the rate of cell production in the crypts at the same site in the same animals; the agreement seen here, and not reported in other investigations, suggests that this method does provide a quantitative method for assessing the absolute rate of cell shedding from the intestinal villus. This argument is based on the assumption that the villus is a constant morphological unit. There is no evidence to suggest that this is not so during the short duration of these experiments, and in the longer term Clarke (1967) has shown that the number of villi in the duodenum of the adult chicken is the same as that in the embryo three days before hatching. The question of a similar constancy in the rat is being investigated.

I thank Dr T. Vickers for encouragement and advice, and for his constructive criticism of the manuscript.

\section{References}

Clarke, R. M. (1967). On the constancy of the number of villi in the duodenum of the post-embryonic domestic fowl. $J$. Embryol. exp. Morph., 17, 131-138.

Clarke, R. M. (1970a). Mucosal architecture and epithelial cell production rate in the small intestine of the albino rat. J. Anat. (Lond.), 107. In press.

Clarke, R. M. (1970b). The effect of starvation upon mucosal architecture and cell production rate in the small intestine of the rat. J. Anat. (Lond.), 107, 384. In press.

Clarke, R. M. (1971). A comparison of metaphase arresting agents and tritiated thymidine autoradiography in measurement of the rate of entry of cells into mitosis in the crypts of Lieberkühn of the rat. In preparation.

Goldsmith, D. P. J. (1969). Measurement of desoxyribonucleic acid (DNA) in canine jejunal fistular fluid. Gut, 10, 497-501.

Leblond, C. P., and Stevens, C. E. (1948). The constant renewal of the intestinal epithelium in the albino rat. Anat. Rec., $100,357-371$.

Loehry, C. A. (1968). The small intestinal mucosa, a stereoscopic study. M.D. dissertation, University of Cambridge.

Loehry, C. A., Croft, D. N., Singh, A. K., and Creamer, B. (1969). Cell turnover in the rat small intestinal mucosa: an appraisal of cell loss. Gut, 10,13-18.

Marmur, J. (1961). A procedure for the isolation of deoxyribonucleic acid from microorganisms. J. molec. Biol., 3, 208-218.

McFarland, J. B., Asplin, A., and Hallaway, H. M. (1968). A new method of studying intestinal mucosal dynamics. Brit. $J$. Surg., 55, 786-789.

O'Connor, T. M. (1966). Cell dynamics in the intestine of the mouse from late fetal life to maturity. Amer. J. Anat., 118, 525-536.

Ringer, S. (1890). Concerning experiments to test the influence of lime, sodium and potassium salts on the development of ova and growth of tadpoles. J. Physiol. (Lona.), 11, 79-84.

Wimber, D. R., and Lamerton, L. F. (1963). Cell population studies on the intestine of continuously irradiated rats. Radiat. Res., 18. 137-146. 